\title{
Electron-Induced State Conversion in Diamond NV Centers Measured with Pump-Probe Cathodoluminescence Spectroscopy
}

\author{
Magdalena Solà-Garcia, ${ }^{* \dagger}{ }^{\dagger}$ Sophie Meuret, ${ }^{\dagger}$ Toon Coenen, ${ }^{\dagger, \S_{\odot}}$ and Albert Polman ${ }^{\dagger \oplus}$ \\ ${ }^{\dagger}$ Center for Nanophotonics, AMOLF, Science Park 104, 1098 XG, Amsterdam, The Netherlands \\ ${ }^{\S}$ Delmic BV, Kanaalweg 4, 2628 EB, Delft, The Netherlands
}

\section{Supporting Information}

ABSTRACT: Nitrogen-vacancy (NV) centers in diamond are reliable single-photon emitters, with applications in quantum technologies and metrology. Two charge states are known for $\mathrm{NV}$ centers, $\mathrm{NV}^{0}$ and $\mathrm{NV}^{-}$, with the latter being mostly studied due to its long electron spin coherence time. Therefore, control over the charge state of the NV centers is essential. However, an understanding of the dynamics between the different states still remains challenging. Here, conversion from $\mathrm{NV}^{-}$to $\mathrm{NV}^{0}$ due to electron-induced carrier generation is shown. Ultrafast pump-probe cathodolumines-

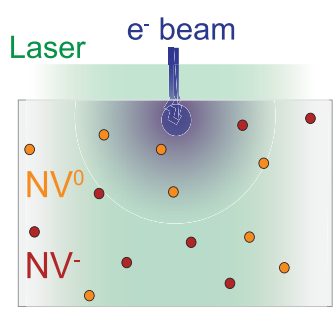

Diamond

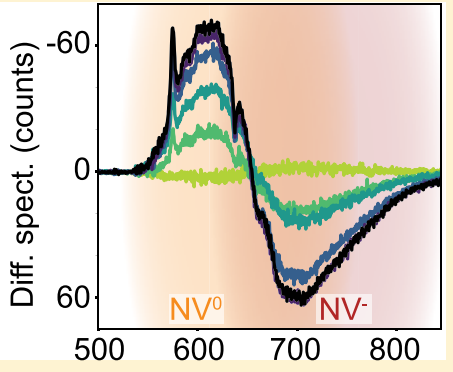
cence spectroscopy is presented for the first time, with electron pulses as pump and laser pulses as probe, to prepare and read out the NV states. The experimental data are explained with a model considering carrier dynamics $(0.8 \mathrm{~ns}), \mathrm{NV}^{0}$ spontaneous emission $(20 \mathrm{~ns})$, and $\mathrm{NV}^{0} \rightarrow \mathrm{NV}^{-}$back transfer $(500$ $\mathrm{ms}$ ). The results provide new insights into the $\mathrm{NV}^{-} \rightarrow \mathrm{NV}^{0}$ conversion dynamics and into the use of pump-probe cathodoluminescence as a nanoscale NV characterization tool.

KEYWORDS: nitrogen-vacancy centers, cathodoluminescence, carrier dynamics, ultrafast electron microscopy, state conversion

$\mathrm{N}$ itrogen-vacancy (NV) centers in diamond are promising elements for quantum optical systems since they are single-photon emitters ${ }^{1,2}$ with high photostability, quantum yield, and brightness, even at room temperature. ${ }^{3-6}$ Moreover, they are integrated inside a wide-bandgap solid-state host, the diamond lattice, making them robust against decoherence and allowing device scalability. ${ }^{7-9} \mathrm{NV}$ centers exhibit two different configurational states, the $\mathrm{NV}^{0}$ state, with a zero-phonon line (ZPL) at $2.156 \mathrm{eV}(\lambda=575 \mathrm{~nm})$, and the $\mathrm{NV}^{-}$state, with a $\mathrm{ZPL}$ at $1.945 \mathrm{eV}(\lambda=637 \mathrm{~nm}){ }^{2} \mathrm{NV}$ centers in the $\mathrm{NV}^{-}$state have received most of the attention in the past years since they exhibit a long electron spin coherence time that can be optically manipulated and read out, ${ }^{9,10}$ which, together with the characteristics mentioned previously, make them suitable as building blocks for quantum technologies, ${ }^{9,11,12}$ nanoscale magnetometry, ${ }^{13,14}$ and other applications. ${ }^{15,16}$ Typically, synthetically prepared diamonds with NV centers contain both $\mathrm{NV}^{0}$ and $\mathrm{NV}^{-}$states. Previous work has shown that the state of an $\mathrm{NV}$ center can be converted from $\mathrm{NV}^{-}$to $\mathrm{NV}^{0}$ (ionization) and vice versa (recombination). For example, the state of the NV centers can be changed by laser irradiation, ${ }^{17-19}$ as well as by shifting the Fermi level, either chemically $^{20-22}$ or by applying an external voltage. ${ }^{23,24}$ Overall, the control and understanding of NV state dynamics is key to the development of efficient quantum optical systems based on NV centers.

So far, most work on NV characterization and state conversion dynamics has focused on optical excitation and readout of the NV state. However, NV centers can also be excited by high-energy (1-200 keV) electrons, using either a scanning or transmission electron microscope (SEM or TEM), while the emitted cathodoluminescence (CL) is collected. Given the small electron beam spot size, the study of NV centers with electron excitation allows for a spatial resolution only limited by the diffusion of carriers, which can be down to the nanometer scale. ${ }^{25}$ This opens the possibility to directly excite NV centers in nanodiamonds with high spatial resolution $^{26}$ and study the coupling of locally excited nanostructures to NV centers, ${ }^{27,28}$ among others. Furthermore, $\mathrm{NV}$ centers are good platforms to study the fundamentals of quantum optics with electrons, in contrast to optical measurements. Electron-beam excitation of NV centers involves a multistep process, in which the primary electron beam inelastically interacts with the diamond lattice, creating bulk plasmons that decay by generating charge carriers. ${ }^{29-31}$ These carriers then diffuse through the diamond and recombine, partially through the excitation of $\mathrm{NV}$ centers. Single-photon emission of individual NV centers excited with electrons has already been demonstrated using measurements of the CL photon autocorrelation function $\left(\mathrm{g}^{(2)}\right) .^{26}$ Interestingly, in CL experiments typically only emission from the $\mathrm{NV}^{0}$ state is observed, ${ }^{25,26,32-37}$ with one exception, ${ }^{32}$ in which a very small $\mathrm{NV}^{-} \mathrm{CL}$ signal was observed at low temperature (16

Received: October 8, 2019

Published: December 2, 2019 
$\mathrm{K})$. This raises the question whether (1) the electron beam does not excite $\mathrm{NV}$ centers in the $\mathrm{NV}^{-}$state, (2) the electron beam quenches the $\mathrm{NV}^{-}$transition, or (3) the electron beam converts $\mathrm{NV}$ centers from the $\mathrm{NV}^{-}$to the $\mathrm{NV}^{0}$ state. Answering this question is essential to understand the $\mathrm{NV}$ state dynamics in general and to further exploit the use of $\mathrm{CL}$ in nanoscale characterization of atomic defects acting as singlephoton emitters.

In this paper we study the interaction of electrons with NV centers, and in particular their state conversion dynamics. We perform the experiments using pump-probe CL spectroscopy, a novel technique that allows studying excited-state dynamics at ultrafast time scales. Previous works combining electron and light excitations in a TEM include photon-induced near-field electron microscopy (PINEM), ${ }^{38,39}$ in which the electron gains or loses energy when interacting with the optically-induced near-field, and femtosecond Lorentz microscopy, ${ }^{40}$ in which the laser-induced magnetization dynamics are probed with the electrons. Similarly, photoinduced carrier dynamics have been studied in an SEM by analyzing the secondary electron yield after laser excitation. ${ }^{41}$ However, in these configurations the electron acts as a probe, since the signal is either transmitted or secondary electrons. In contrast, in pump-probe CL the final signal is the emitted light, either CL or photoluminescence (PL); therefore, the electron can also act as a pump. In this work, we use an ultrafast SEM in which picosecond electron pulses are used to pump the diamond sample, while synchronously we optically probe the NV state. The electron pulses are generated using a laser-driven cathode configuration, a technique initially demonstrated by Merano et al. using a gold cathode ${ }^{42}$ and further developed in combination with field-emission guns (FEGs) to improve the spatial and temporal resolution. ${ }^{43,44}$ After ultrafast excitation of the $\mathrm{NV}$ centers, the CL and PL spectra are collected for spectral and temporal characterization. We find that repeated pulsed electron excitation $(5.04 \mathrm{MHz})$ causes a state conversion from $\mathrm{NV}^{-}$to $\mathrm{NV}^{0}$, until a steady state is achieved in which the electron-induced $\mathrm{NV}^{-} \rightarrow \mathrm{NV}^{0}$ conversion is balanced by the reverse $\mathrm{NV}^{0} \rightarrow \mathrm{NV}^{-}$back transfer. The steady-state $\mathrm{NV}^{0}$ population under electron irradiation can be controlled by the number of electrons per pulse. We describe the results with a model that includes electron-induced carrier generation and diffusion, with the NV centers acting as carrier traps and electrons converting $\mathrm{NV}$ centers from the $\mathrm{NV}^{-}$to the $\mathrm{NV}^{0}$ state. The time dynamics of carrier diffusion $(\sim 0.8 \mathrm{~ns}), \mathrm{NV}^{0}$ decay $(\sim 20 \mathrm{~ns})$, and $\mathrm{NV}^{0} \rightarrow \mathrm{NV}^{-}$back transfer $(\sim 500 \mathrm{~ms})$ are clearly observed from the pump-probe transients.

\section{PUMP_PROBE CL SETUP}

The pump-probe CL experiments are performed inside a SEM. We focus the fourth harmonic $(\lambda=258 \mathrm{~nm})$ of an $\mathrm{Yb}$ doped fiber fs laser on the electron gun to generate electron pulses by photoemission ${ }^{42,45}$ (Figure 1a). Photoemission of electron pulses using this setup was characterized previously, ${ }^{46}$ showing that the generated electron pulses are in the picosecond regime, similar to other work. ${ }^{44,47}$ The electron beam is focused on a single spot on the sample, corresponding to the center of the area irradiated by the laser beam. We synchronously excite the sample at the electron-irradiated region with second-harmonic $(\lambda=517 \mathrm{~nm})$ pulses generated by the same fs laser, which are focused inside the SEM chamber to a $\sim 10 \mu \mathrm{m}$ diameter spot on the sample using an $\mathrm{Al}$ parabolic mirror. The second harmonic path length can be
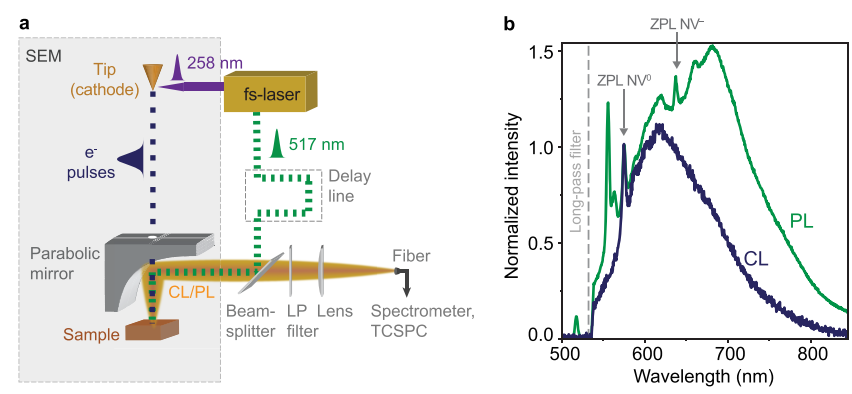

Figure 1. Pump-probe CL setup and NV center spectra. (a) Schematic of the pump-probe CL setup. The fourth harmonic $(\lambda=$ $258 \mathrm{~nm}$ ) of a fs laser is focused on the electron cathode to induce photoemission of electron pulses $(0-400$ electrons/pulse, picosecond temporal spread). The second harmonic $(\lambda=517 \mathrm{~nm})$ of the same laser synchronously excites the sample to read out the NV state. The light pulse is delayed $1.3 \mathrm{~ns}$ with respect to the electron pulse. The emitted light, CL, PL, or both, is collected using a parabolic mirror and analyzed with a spectrometer or TCSPC module. A long-pass (LP, $\lambda>532 \mathrm{~nm}$ ) filter is used to remove the light from the excitation laser. (b) Photoluminescence (green) and cathodoluminescence (blue) spectra obtained independently when exciting a bulk diamond sample with either a $517 \mathrm{~nm}$ pulsed laser beam $(0.9 \mathrm{~nJ} /$ pulse $)$ or a 5 $\mathrm{keV}$ pulsed electron beam (400 electrons/pulse), respectively. Both spectra are obtained when exciting with a repetition rate of $5.04 \mathrm{MHz}$ and at the same position on the sample. CL and PL spectra have been normalized by the amplitude of the $\mathrm{NV}^{0} \mathrm{ZPL}$ at $575 \mathrm{~nm}$.

tuned within a \pm 2 ns time window, such that the optical excitation pulse on the sample is delayed (or advanced) with respect to the electron pulse. CL and PL are collected by the parabolic mirror and directed to either a spectrometer or a time-correlated single photon counting (TCSPC) module. We use a $300 \mu \mathrm{m}$ thick single-crystal diamond sample (obtained from Element 6 Inc.), grown by chemical-vapor deposition $(<1$ ppm nitrogen concentration, $<0.05 \mathrm{ppm}$ boron concentration), containing an approximate $\mathrm{NV}$ concentration of $\left[\mathrm{NV}_{\text {tot }}\right]=1.2$ $\mathrm{ppb}\left(200 \mu \mathrm{m}^{-3}\right)$. The sample is coated with a thin charge dissipation layer (E-spacer 300) to avoid charging when exciting with the electron pulses.

\section{$\mathrm{CL}$, PL, AND PUMP-PROBE MEASUREMENTS}

Using the pump-probe CL setup, we acquire first PL and CL spectra, shown in Figure $1 \mathrm{~b}$. The PL spectrum shows emission from the ZPL of $\mathrm{NV}^{-}(\lambda=637 \mathrm{~nm})$ and $\mathrm{NV}^{0}(\lambda=575 \mathrm{~nm})$, with both ZPL transitions accompanied by phonon replicas, forming a broadband spectrum in the $575-800 \mathrm{~nm}$ spectral range. A Raman peak at $\lambda=555 \mathrm{~nm}$ is also observed, ${ }^{48}$ as well as a peak around $563 \mathrm{~nm}$, which has been observed in previous work and preliminarily attributed to a divacancy defect. ${ }^{37,49,50}$ The CL spectrum, obtained when exciting with a $5 \mathrm{keV}$ pulsed electron beam, clearly shows the ZPL of the $\mathrm{NV}^{0}$ state, with phonon sideband, but no emission from the $\mathrm{NV}^{-}$state is observed, similar to previous work..$^{25,26,32-37}$ The relative contribution of $\mathrm{NV}^{-}$and $\mathrm{NV}^{0}$ states to the PL spectrum is obtained by a fitting procedure, with the CL spectrum as a reference for the spectral shape of the $\mathrm{NV}^{0}$ emission (see Supporting Information). Using estimated optical absorption cross sections at the laser excitation wavelength (see Supporting Information) we derive the $\mathrm{NV}^{-}$and $\mathrm{NV}^{0}$ fractions: $\left[\mathrm{NV}^{-}\right] /\left[\mathrm{NV}_{\text {tot }}\right] \approx 0.4$ and $\left[\mathrm{NV}^{0}\right] /\left[\mathrm{NV}_{\text {tot }}\right] \approx 0.6$.

Our pump-probe measurements consist of the independent acquisition of a set of spectra: only CL, only PL, and pumpprobe (PP). The latter is obtained under simultaneous electron 

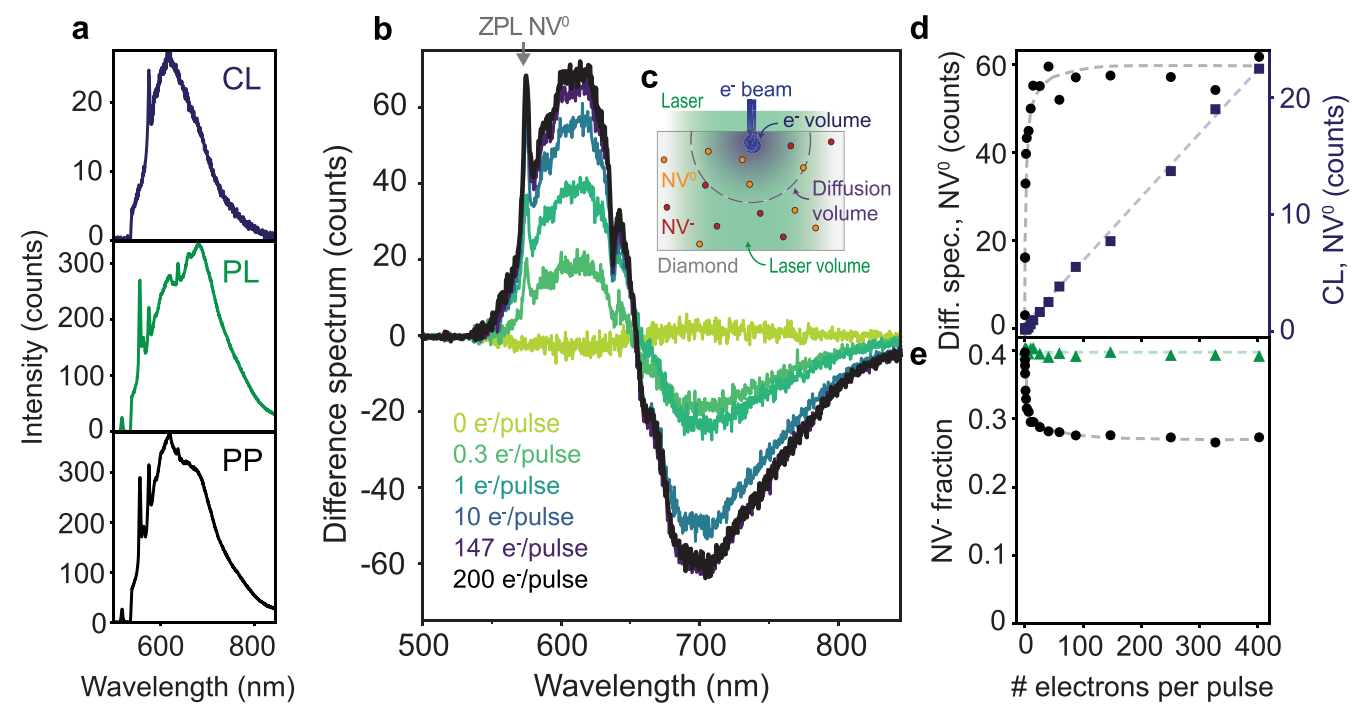

Figure 2. $\mathrm{NV}^{-} \rightarrow \mathrm{NV}^{0}$ conversion under electron excitation (a) Top: CL spectrum ( $5 \mathrm{keV}, 400$ electrons/pulse), middle: PL spectrum $(\lambda=517$ $\mathrm{nm}, 0.9 \mathrm{~nJ} /$ pulse), bottom: pump-probe (PP) spectrum obtained when both electrons and light (same conditions as before) excite the sample $(5.04 \mathrm{MHz}$ ). The acquisition time was $1 \mathrm{~min}$ in all cases. (b) Difference spectrum, obtained by subtracting CL and PL spectra from the PP spectrum. (c) Sketch of the laser and electron excitation on the sample, representing the different volumes of primary electron interaction, diffusion of carriers, and laser volume. (d) NV $\mathrm{NPL}^{0}$ intensity $(\lambda=575 \mathrm{~nm}$ ) of the difference spectrum (black circles) and from the CL-only spectrum (blue squares) as a function of the average number of electrons per pulse. The $\mathrm{NV}^{0} \mathrm{ZPL}$ of the difference spectrum shows saturation at around 20 electrons/pulse, while in the case of CL the dependence is linear. Dashed lines are shown as guides for the eye. (e) $\mathrm{NV}^{-}$fraction obtained from the $\mathrm{PP}$ as a function of the number of electrons per pulse (black circles). The green triangles indicate the $\mathrm{NV}^{-}$fraction derived from the PL spectra (all at the same PL pump power). Dashed lines are guides for the eye.

and light excitation, with the light pulse arriving $1.3 \mathrm{~ns}$ after each electron pulse. A set of spectra is shown in Figure 2a. All measurements were performed at the same spot on the sample, to avoid effects due to concentration inhomogeneities. In addition to the differences in the $\mathrm{PL}$ and $\mathrm{CL}$ spectra mentioned above, we also observe that the PL signal is an order of magnitude higher than the CL one. Even though a detailed comparison between both magnitudes is complex due to the different incident powers and excitation mechanisms, we can estimate the number of NVs excited in each case. The laser spot size has a diameter of around $10 \mu \mathrm{m}$ and large penetration depth, due to the low absorption of diamond and low NV concentration. Therefore, the volume is mostly determined by the collection volume of the setup (see Methods). The primary interaction volume of the $5 \mathrm{keV}$ electron beam is around 0.4 $\mu \mathrm{m}^{3}$, as calculated from Monte Carlo-based simulations using the software Casino. ${ }^{51}$ Even though the effective volume is enlarged due to carrier diffusion, as will be shown below, it is still smaller than the volume excited by the laser. A sketch of both volumes is shown in Figure 2c. Taking into account the optical cross sections and collection geometry, we estimate that we collect PL from around $1.4 \times 10^{4} \mathrm{NVs}$ per pulse for an incident power of $0.9 \mathrm{~nJ}$ (per pulse). Comparing the magnitude of the PL and CL signals, we can also extract that an average of $900 \mathrm{NV}$ centers in the $\mathrm{NV}^{0}$ state are excited per electron pulse, in the steady-state situation, as will be discussed further on. In this case, each electron pulse contained 400 electrons with $5 \mathrm{keV}$ energy (corresponding to $0.32 \mathrm{pJ}$ per pulse).

Using the PL, CL, and PP spectra shown above, we can analyze the effect of electron irradiation on $\mathrm{NV}$ centers. We define the quantity of a difference spectrum, obtained when subtracting CL and PL spectra from the PP spectrum. This analysis allows to study the correlation between electron and light excitation of the NV centers. Therefore, no correlation would lead to a flat difference spectrum. Instead, the difference spectrum obtained from the data in Figure 2a exhibits clear features, as shown in Figure $2 b$ (black curve). We observe an increase of the signal (positive counts) in the lower-wavelength spectral band, corresponding to the $\mathrm{NV}^{0}$ emission. As a reference, we observe a clear peak corresponding to the $\mathrm{NV}^{0}$ ZPL. We also observe a concomitant decrease in the longerwavelength band, corresponding to $\mathrm{NV}^{-}$emission. In this case, the $\mathrm{NV}^{-} \mathrm{ZPL}$ is visible as a dip. This implies that after electron excitation the number of emitting $\mathrm{NV}^{0}$ centers is increased, while the number of $\mathrm{NV}^{-}$centers is decreased. The results suggest that centers in the $\mathrm{NV}^{-}$states are converted into $\mathrm{NV}^{0}$ states under electron irradiation, corresponding to hypothesis (3) exposed earlier in the text. Difference spectra derived for different sets of measurements at $0.3,1,10$, and 147 electrons per pulse are also shown in Figure $2 b$, as well as a reference measurement (no electron irradiation). Each set of measurements corresponds to the acquisition of independent CL, PL, and PP spectra, in which the number of electrons per pulse is varied, while keeping the laser excitation power constant at 0.9 $\mathrm{nJ}$ per pulse. We again observe $\mathrm{NV}^{-} \rightarrow \mathrm{NV}^{0}$ conversion, with the number of converted centers rising for increasing average number of electrons per pulse. This behavior in the difference spectra was consistently observed in other measurements at different areas of the sample and also with other electron energies (30 keV, Figure S1).

To further investigate the electron-induced $\mathrm{NV}^{-} \rightarrow \mathrm{NV}^{0}$ conversion trend, we plot the amplitude of the $\mathrm{NV}^{0} \mathrm{ZPL}$ as a function of the number of electrons per pulse (Figure 2d). Saturation of the signal from the $\mathrm{NV}^{0} \mathrm{ZPL}$ is observed above $\sim 20$ electrons per pulse, suggesting that this is the required electron flux (at $5.04 \mathrm{MHz}$ ) to induce the saturation of the $\mathrm{NV}^{-}$conversion in the volume of the sample excited by electrons. For reference, Figure $2 \mathrm{~d}$ also shows the CL intensity for the $\mathrm{NV}^{0} \mathrm{ZPL}$ as a function of the number of electrons per 
pulse. The plot shows a linear trend, indicating that the $\mathrm{NV}^{0}$ CL signal is not saturating with increasing electron dose; that is, there is no strong depletion of the ground-state population. Therefore, from these results we derive that electrons can either excite $\mathrm{NV}$ centers in the $\mathrm{NV}^{0}$ state, which leads to a linear dependence on the electron flux, or convert $\mathrm{NV}^{-}$into $\mathrm{NV}^{0}$, which saturates with increasing number of electrons per pulse.

From the data in Figure $2 \mathrm{~b}$ we can also derive the $\mathrm{NV}^{-}$ population as a function of the number of electrons per pulse, as plotted in Figure 2e. This derivation is done by fitting the $\mathrm{NV}^{0}$ and $\mathrm{NV}^{-}$contributions from the PP measurements (see Supporting Information). Starting from the initial $\mathrm{NV}^{-}$fraction of 0.4 for the reference measurement, as already derived before, the population of centers in the $\mathrm{NV}^{-}$state rapidly decreases with increasing number of electrons per pulse, reaching a saturation level corresponding to a $0.26 \mathrm{NV}^{-}$ fraction. We attribute this saturation level to the full conversion of $\mathrm{NV}^{-}$centers into $\mathrm{NV}^{0}$ centers within the volume excited by the electrons, as will be discussed further on. The fact that the $\mathrm{NV}^{-}$fraction does not reach zero at saturation is attributed to the difference between excitation and collection volumes of electron and laser beam, as sketched in Figure 2c. For completeness, in Figure 2e we also show the $\mathrm{NV}^{-}$fraction derived from the PL measurements taken in each set of measurements from Figure $2 \mathrm{~b}$. We observe that the $\mathrm{NV}^{-}$ fraction under only laser irradiation remains approximately constant, meaning that the $\mathrm{NV}^{-}$population before each set of measurements is identical. The fact that the $\mathrm{NV}^{-}$population is unchanged also implies that the electron-induced $\mathrm{NV}^{-} \rightarrow \mathrm{NV}^{0}$ conversion is reversible, i.e., there is an $\mathrm{NV}^{0} \rightarrow \mathrm{NV}^{-}$back transfer process, and that damage induced by the electron to the sample is negligible. Given that $\mathrm{NV}^{-} \leftrightarrow \mathrm{NV}^{0}$ conversion has also been observed due only to laser irradiation, ${ }^{17-19}$ we also acquired PL spectra at different incident powers. The results are presented in Figure $\mathrm{S} 2$ and show that the $\mathrm{NV}^{-}$ fraction remains constant for increasing laser power, therefore proving that $\mathrm{NV}$ conversion due to only laser irradiation is negligible in our experiment. Pump-probe measurements with different delays between electron and light were also acquired (Figure S3), but no significant differences are observed. This is attributed to the fact that the $\mathrm{NV}^{0} \rightarrow \mathrm{NV}^{-}$back transfer is on the order of milliseconds, as will be demonstrated below, larger than the time between pulses (198 ns at $5.04 \mathrm{MHz}$ ).

\section{EXCITATION, EMISSION, AND CONVERSION DYNAMICS}

In order to further describe the interaction of electrons with $\mathrm{NV}$ centers, we study the excitation and emission dynamics of $\mathrm{NV}$ centers at the nanosecond time scale, as well as the $\mathrm{NV}^{0} \rightarrow$ $\mathrm{NV}^{-}$back transfer that occurs in the millisecond scale. The time-dependent CL emission from NV centers upon electron excitation is shown in Figure 3a, which has been measured using the TCSPC technique. Notice that the CL intensity corresponds only to emission from excited $\mathrm{NV}^{0}$ centers, given that $\mathrm{NV}^{-}$emission is not probed with $\mathrm{CL}$. The $\mathrm{CL}$ signal exhibits a gradual increase in the first 2 ns, reaching a maximum emission at around $2.2 \mathrm{~ns}$ (see inset). We ascribe this initial increase to the diffusion of carriers beyond the primary electron-excited volume, which increases the excited $\mathrm{NV}^{0}$ population well after the initial ps-electron pulse excitation. After the first 2 ns we observe a decay of the CL intensity, from which we extract a characteristic decay time of

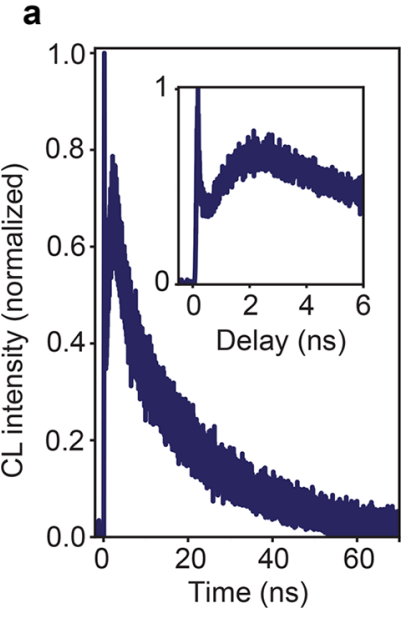

b

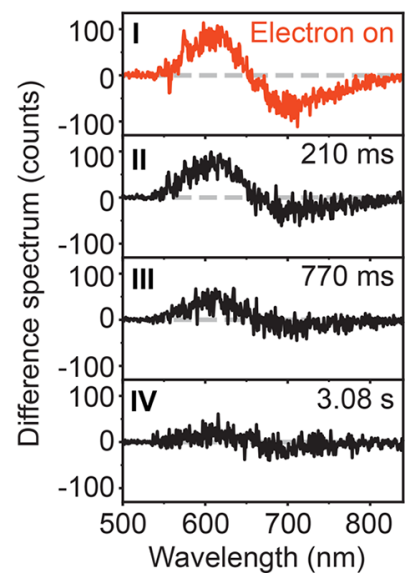

Figure 3. Carrier diffusion, excitation, and back transfer dynamics. (a) Peak-normalized CL intensity upon pulsed electron excitation $(5 \mathrm{keV}$, $\sim 450$ electrons/pulse, $5.04 \mathrm{MHz}$ ) at $t=0 \mathrm{~ns}$, measured with timecorrelated single-photon counting. Data are taken in the $\mathrm{NV}^{0} 575-$ $725 \mathrm{~nm}$ spectral band. Inset: enlarged early time scale. (b) Difference spectrum (defined as PP-CL-PL) obtained with the electron beam on (I) and $210 \mathrm{~ms}, 770 \mathrm{~ms}$, and $3.08 \mathrm{~s}$ after the electron beam was blanked (II-IV, respectively). The $\mathrm{NV}^{0} \rightarrow \mathrm{NV}^{-}$back transfer takes around $500 \mathrm{~ms}$. The time resolution of this experiment is $70 \mathrm{~ms}$.

$\sim 20 \mathrm{~ns}$, in agreement with the typical radiative decay time of excited $\mathrm{NV}^{0}$ centers. $^{26,52}$ We also observe a $\sim 100$ ps spike at 0 ns, which accounts for around $1 \%$ of the total intensity. The origin of this fast decay is unknown. The intensity of this peak depends on the position on the sample, as well as electron energy. Nevertheless, the amplitude of this peak does not show any correlation with the magnitude of the $\mathrm{NV}^{-} \rightarrow \mathrm{NV}^{0}$ conversion, from which we infer that both effects are unrelated.

In contrast to the fast carrier diffusion and $\mathrm{NV}^{0}$ emission dynamics, previous studies of optically induced $\mathrm{NV}^{0} \leftrightarrow \mathrm{NV}^{-}$ conversion suggest that the $\mathrm{NV}^{0} \rightarrow \mathrm{NV}^{-}$back transfer is in the millisecond regime. ${ }^{17}$ To study this, we performed timeresolved spectral measurements over a millisecond time scale. We used the minimum exposure time possible in our spectrometer, acquiring a spectrum every $70 \mathrm{~ms}$. The repetition rate is kept at $5.04 \mathrm{MHz}$, as in the previous experiments. We performed a spectral acquisition sequence in which initially both the electron and laser beam were irradiating the sample (PP spectrum). At some point during the acquisition, the electron beam was blanked, while the laser continued exciting the sample, and spectra kept being collected every $70 \mathrm{~ms}$. In this way, the NV population can be probed immediately after the electron beam is switched off. Afterward, we also acquired CL and PL spectra with the same exposure time, such that a difference spectrum can be derived, similar to Figure $2 \mathrm{~b}$. An example of the obtained difference spectrum is shown in Figure 3b-I, which again reflects the $\mathrm{NV}^{-} \rightarrow \mathrm{NV}^{0}$ conversion by the electron-excited carriers. In this case, the electron beam was still irradiating the sample. Figure $3 \mathrm{~b}-\mathrm{II}$ shows the difference spectrum obtained $210 \mathrm{~ms}$ after switching off the electron beam. Notice that here the difference spectrum is obtained by subtracting only PL from the PP measurement, given that there is no CL. We observe a $30 \%$ decrease of the intensity of the difference spectrum, indicating that most of the converted $\mathrm{NV}^{-}$centers still remain in the $\mathrm{NV}^{0}$ state, and only some have converted back into $\mathrm{NV}^{-}$. Results after $770 \mathrm{~ms}$ and $3.08 \mathrm{~s}$ are also plotted (Figure $3 \mathrm{~b}-\mathrm{III}, \mathrm{IV}$ ), in which we observe 
a progressive decay of the signal, indicating that $\mathrm{NV}^{0}$ centers are converted back to the $\mathrm{NV}^{-}$state. A complete transient of the average signal in the difference spectrum as a function of time is provided in Figure S4. These data indicate that the electron-induced $\mathrm{NV}^{-} \rightarrow \mathrm{NV}^{0}$ state conversion is reversible, with the back transfer taking place within a characteristic time of $\sim 500 \mathrm{~ms}$. This time scale is in agreement with earlier work, in which back transfer of optically induced $\mathrm{NV}^{-} \rightarrow \mathrm{NV}^{0}$ conversion was found to occur with a characteristic time of 465 ms. $^{17}$

\section{DISCUSSION AND PHENOMENOLOGICAL MODEL}

Optically induced state conversion from $\mathrm{NV}^{-}$to $\mathrm{NV}^{0}$ has been previously explained to take place by the release of an electron from the $\mathrm{NV}^{-}$center to the conduction band of diamond. ${ }^{19,52-54}$ Literature values for the difference in energy between the $\mathrm{NV}^{-}$ground state and the conduction band range from 2.6 to $4.3 \mathrm{eV},{ }^{17,19,52}$ and the $\mathrm{NV}^{-} / \mathrm{NV}^{0}$ optical conversion typically requires a two-photon absorption process. In our experiment, we propose a model in which electron-hole pairs generated from the electron cascade can recombine, thus providing the energy to induce the release of the bound electron from the $\mathrm{NV}^{-}$center, given that the bandgap of diamond is $5.5 \mathrm{eV}$. This conversion mechanism is similar to that in optical experiments, with the difference that the energy is provided by a carrier recombination event instead of two pump photons. This model is in agreement with previous work in which emission only from the $\mathrm{NV}^{0}$ state was observed when exciting with far-UV photons $(\lambda=170 \mathrm{~nm}$, above the bandgap of diamond $)^{55}$ and in electroluminescence. ${ }^{56,57}$ In both cases, charge carriers are generated and NV centers are excited through the recombination of carriers, similar to CL. In addition to this, the energy provided by a single carrier recombination event is larger than the energy needed to induce the $\mathrm{NV}^{-} \rightarrow \mathrm{NV}^{0}$ conversion, suggesting that a single carrier recombination event could already release the electron, without the need to first excite the $\mathrm{NV}^{-}$center as in the case of optical experiments. ${ }^{19,52-54}$ The latter suggestion requires further studies in the mechanism of $\mathrm{NV}^{-} \rightarrow \mathrm{NV}^{0}$ conversion by carrier recombination, which are beyond the scope of this paper.

To qualitatively analyze the data shown above, we model the electron-induced $\mathrm{NV}^{-} \rightarrow \mathrm{NV}^{0}$ state conversion by means of a three-dimensional model, considering carrier diffusion and $\mathrm{NV}$ center conversion and excitation. We start by modeling the dynamics in the nanosecond regime, corresponding to carrier diffusion and $\mathrm{NV}^{0}$ decay. We use Monte Carlo simulations, using the software Casino, ${ }^{51}$ to obtain the three-dimensional spatial distribution of inelastic scattering events of the primary $5 \mathrm{keV}$ electron beam. Most of the energy lost by the electron corresponds to the generation of bulk plasmons, described as excitations of the outer-shell electrons, ${ }^{29}$ with an energy corresponding to $31 \mathrm{eV}$ for diamond. ${ }^{30}$ We then model the initial carrier distribution with a 3D Gaussian distribution, with standard deviation $\sigma=0.185 \mu \mathrm{m}$ estimated from the plasmon distribution derived from Casino simulations and amplitude proportional to the number of electrons per pulse. We assume that each bulk plasmon effectively generates an average of two electron-hole pairs. ${ }^{30}$ The concentration of charge carriers as a function of time and space $\left(\rho_{\mathrm{eh}}(r, t)\right)$ is then obtained by solving the diffusion equation, with carrier recombination described with a lifetime $\tau_{\mathrm{R}}$.
Taking into account carrier diffusion, we model the concentration of $\mathrm{NV}^{-}$in the ground state $\left(\rho_{-}\right)$and $\mathrm{NV}^{0}$ in the ground $\left(\rho_{0}^{\mathrm{g}}\right)$ and excited $\left(\rho_{0}^{\mathrm{e}}\right)$ states by means of a rate equation model:

$$
\frac{\partial \rho_{-}(r, t)}{\partial t}=-v_{\mathrm{th}} \rho_{\mathrm{eh}}(r, t) \sigma_{\mathrm{c}}^{\mathrm{eh}} \rho_{-}(r, t)+\frac{\rho_{-\mathrm{i}}-\rho_{-}(r, t)}{\tau_{\text {back }}}
$$

$$
\begin{aligned}
\frac{\partial \rho_{0}^{\mathrm{g}}(r, t)}{\partial t}= & v_{\mathrm{th}} \rho_{\mathrm{eh}}(r, t)\left[\sigma_{\mathrm{c}}^{\mathrm{eh}} \rho_{-}(r, t)-\sigma_{0}^{\mathrm{eh}} \rho_{0}^{\mathrm{g}}(r, t)\right] \\
& +\frac{\rho_{0}^{\mathrm{e}}(r, t)}{\tau_{0}}-\frac{\rho_{-\mathrm{i}}-\rho_{-}(r, t)}{\tau_{\text {back }}}
\end{aligned}
$$

$$
\frac{\partial \rho_{0}^{\mathrm{e}}(r, t)}{\partial t}=v_{\mathrm{th}} \rho_{\mathrm{eh}}(r, t) \sigma_{0}^{\mathrm{eh}} \rho_{0}^{\mathrm{g}}(r, t)-\frac{\rho_{0}^{\mathrm{e}}(r, t)}{\tau_{0}}
$$

where $v_{\text {th }}$ is the thermal velocity of carriers, $\sigma_{0}^{\text {eh }}$ is the crosssection to excite $\mathrm{NV}^{0}$ states by carriers, $\sigma_{\mathrm{c}}^{\mathrm{eh}}$ is the $\mathrm{NV}^{-} \rightarrow \mathrm{NV}^{0}$ conversion cross-section, $\tau_{0}$ is the lifetime of the excited $\mathrm{NV}^{0}$ state, $\tau_{\text {back }}$ accounts for the $\mathrm{NV}^{0} \rightarrow \mathrm{NV}^{-}$back transfer, and $\rho_{-\mathrm{i}}$ is the initial uniform concentration of $\mathrm{NV}^{-}$. In this model we assume that $\mathrm{NV}^{0}$ states can be excited by carriers, but $\mathrm{NV}^{-}$ states cannot, given that we do not observe $\mathrm{NV}^{-}$signal in the CL measurements. Moreover, the interaction of the primary electron beam (picosecond temporal spread) with the sample, including generation of bulk plasmons and decay into carriers, is treated as instantaneous, given that it is much shorter than the characteristic time scale of the dynamics in eqs $1 \mathrm{a}-1 \mathrm{c}$.

Numerically solving the system of differential equations over time, and integrating $\rho_{0}^{\mathrm{e}}(r, t)$ over the collection volume, allows to fit the trend in the first $2 \mathrm{~ns}$ of the time-dependent CL intensity shown in Figure 3a. The carrier lifetime derived from the fit is $\tau_{\mathrm{R}}=0.8 \mathrm{~ns}$, corresponding to a diffusion length of 0.9 $\mu \mathrm{m}$, which is in agreement with values reported for samples with a similar concentration of NV centers. ${ }^{58}$ From the model we also find that excitation with 400 electrons $(5 \mathrm{keV})$ leads to about $740 \mathrm{NV}^{0}$ centers excited per pulse, close to the value independently derived from the comparison of $\mathrm{PL}$ and $\mathrm{CL}$ intensities in Figure 2a,b. Taking into account the obtained carrier lifetime, in Figure $4 a$ we plot the spatial distribution of the carrier concentration at $t=0 \mathrm{~ns}$ (solid black) and after 1 and $5 \mathrm{~ns}$ (dashed dark green and dotted light green, respectively), obtained from the expression of $\rho_{\mathrm{eh}}(r, t)$ (eq S1). The carrier distribution rapidly spreads out due to diffusion, with the total amount of carriers decreasing as a result of carrier recombination.

The calculated spatial distribution of the $\mathrm{NV}^{-}$concentration is shown in Figure $4 \mathrm{~b}$, again at $t=0,1$, and $5 \mathrm{~ns}$, obtained by solving eqs $1 \mathrm{a}-1 \mathrm{c}$. Given that the electron excitation crosssections for $\mathrm{NV}^{0}$ excitation and $\mathrm{NV}^{-} \rightarrow \mathrm{NV}^{0}$ conversion are unknown, we estimate them by considering the known exciton capture cross-section of a nitrogen impurity in diamond, ${ }^{59} \sigma_{0}^{\text {eh }}$ $=\sigma_{c}^{\text {eh }}=3 \times 10^{-6} \mu \mathrm{m}^{2}$. We consider $v_{\text {th }}=100 \mu \mathrm{m} / \mathrm{ns}, \tau_{\text {back }}=$ $500 \mathrm{~ms}$, as obtained from the experimental data in Figure $3 \mathrm{~b}$, and an initial homogeneous $\mathrm{NV}^{-}$fraction of 0.4 (black line in Figure $4 \mathrm{~b}$ for $t=0 \mathrm{~ns}$ ), corresponding to the experimental data in Figure 2e. We observe that $1 \mathrm{~ns}$ after the first pulse NV centers in the $\mathrm{NV}^{-}$state that are located within a $1 \mu \mathrm{m}$ range from the initial electron cascade have been converted to $\mathrm{NV}^{0}$ due to the interaction with carriers. For larger times ( $5 \mathrm{~ns})$ the 

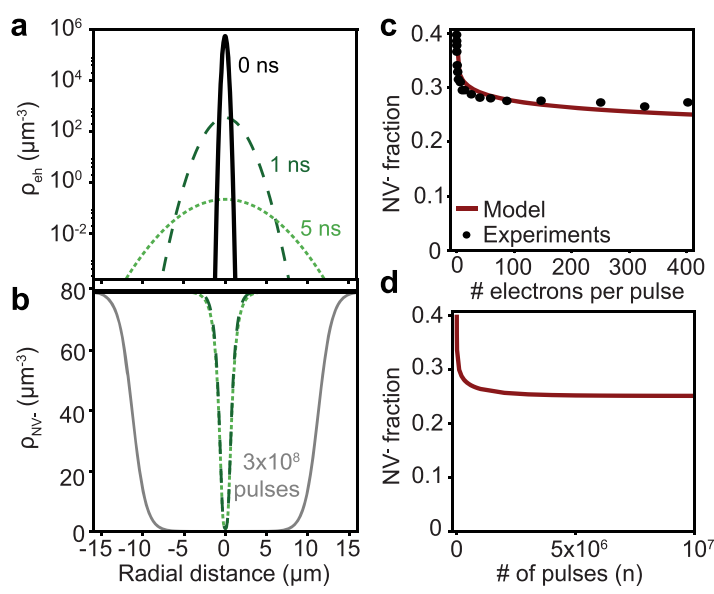

Figure 4. Carrier diffusion and rate equation models. (a) Spatial distribution of the concentration of carriers at $t=0,1$, and 5 ns (solid black, dashed dark green, and dotted light green, respectively). (b) Initial spatial distribution of the concentration of $\mathrm{NV}^{-}$states (solid black) and after 1 and 5 ns (dashed dark and dotted light green) after a single electron pulse. The spatial distribution of $\mathrm{NV}^{-}$states after $3 \times$ $10^{8}$ pulses, corresponding to a typical acquisition time $(\sim 1 \mathrm{~min})$, is also plotted (solid gray). (c) Modeled $\mathrm{NV}^{-}$fraction as a function of the number of electrons per pulse (dark red curve), together with the experimental data (black circles). (d) $\mathrm{NV}^{-}$fraction as a function of the number of pulses (400 electrons/pulse), obtained using the discrete rate equation model.

distribution of converted $\mathrm{NV}^{-}$centers is nearly the same as for $t=1 \mathrm{~ns}$, as nearly all carriers have recombined.

In order to account for longer time scales, corresponding to the back transfer from $\mathrm{NV}^{0}$ to $\mathrm{NV}^{-}$and the time of acquisition of our experiments (typically $1 \mathrm{~min}, \sim 3 \times 10^{8}$ pulses), we developed a discrete rate equation model. In this case, the concentration of $\mathrm{NV}^{-}$centers is modeled as a function of the pulse number $(n)$ :

$$
\rho_{-}(r, n)=\rho_{-}(r, 0) \frac{\beta+\alpha(r)[1-\alpha(r)-\beta]^{n}}{\alpha(r)+\beta}
$$

where

$$
\alpha(r)=1-\mathrm{e}^{-v_{\mathrm{th}} \sigma_{\mathrm{c}} \int_{0}^{T} \rho_{\mathrm{eh}}(r, t) \mathrm{d} t}
$$

is the probability of carrier-induced conversion of centers in the $\mathrm{NV}^{-}$states between subsequent pulses, with $T$ being the time between pulses (198 ns at $5.04 \mathrm{MHz}$ ), and

$$
\beta=1-\mathrm{e}^{-T / \tau_{\text {back }}}
$$

is the probability that an $\mathrm{NV}^{0}$ center transfers back to the $\mathrm{NV}^{-}$ state, again between subsequent pulses (see Supporting Information). Using this model, in Figure $4 \mathrm{~b}$ we plot the spatial distribution of $\mathrm{NV}^{-}$centers after $3 \times 10^{8}$ pulses (solid gray), corresponding to a typical acquisition time (1 $\mathrm{min}$ ), in which the steady state has been reached. The calculated steadystate $\mathrm{NV}^{-}$fraction as a function of the number of electrons per pulse is shown in Figure $4 c$, which is overlaid with the experimental data from Figure 2e (black circles). Each point in the plot corresponds to the steady-state value calculated using eq 2 and integrated over the excitation and collection volume (see Supporting Information). In our model, taking the parameters discussed above, the only fit parameter is the collection depth of the CL system, which is $23 \mu \mathrm{m}$ for the best fit. This is a reasonable value given the confocal geometry of the CL/PL collection system (see Methods). Figure 4d shows the calculated $\mathrm{NV}^{-}$fraction as a function of the number of pulses. We observe that the $\mathrm{NV}^{-}$fraction saturates for $\sim 5 \times$ $10^{6}$ pulses $(1 \mathrm{~s})$, consistent with the fact that the steady state is reached for a time longer than the $\mathrm{NV}^{0} \rightarrow \mathrm{NV}^{-}$back transfer time. Overall, the model qualitatively describes properly the experimental data, therefore giving further proof for the proposed electron-induced mechanism for $\mathrm{NV}^{-} \rightarrow \mathrm{NV}^{0}$ conversion dynamics.

\section{CONCLUSIONS}

In conclusion, we have used pump-probe CL spectroscopy to show that high-energy $(5 \mathrm{keV})$ electron irradiation of $\mathrm{NV}$ centers induces a state conversion from the $\mathrm{NV}^{-}$to the $\mathrm{NV}^{0}$ state. We show that the $\mathrm{NV}^{-}$population decreases when increasing the number of electrons per pulse that excite the sample, until saturation is reached, which is attributed to the full conversion of the $\mathrm{NV}^{-}$centers in the volume excited through the electrons. Experiments also show that the $\mathrm{NV}^{-} \rightarrow$ $\mathrm{NV}^{0}$ conversion is reversible, with a typical back transfer time of $500 \mathrm{~ms}$. We present a three-dimensional rate equation model, considering diffusion of electron-generated charge carriers and taking into account the integrated effect of subsequent pulses, which qualitatively describes the experimental results. This work shows that $\mathrm{NV}^{-}$centers are effectively converted to $\mathrm{NV}^{0}$ centers by electron irradiation and explains why $\mathrm{NV}^{-}$emission is not observed in CL measurements. We envision that the pump-probe $\mathrm{CL}$ approach presented in this work can be applied to other complex solid-state emitter systems, to obtain further insight in their complex dynamical behavior.

\section{METHODS}

Ultrafast SEM. A schematic of the setup is shown in Figure 1a. The pump-probe CL experiments are performed inside an SEM (Thermo Fisher Scientific/XL30 FEI) containing a Schottky field-emission electron cathode consisting of a $\mathrm{ZrO}$ coated $\mathrm{W}$ tip. The conditions used to generate the electron pulses are discussed in ref 46. We use a diode-pumped $\mathrm{Yb}$ doped fiber system (IMPULSE Clark-MXR) providing $250 \mathrm{fs}$ light pulses at a wavelength of $\lambda=1035 \mathrm{~nm}$ and repetition rate of $5.04 \mathrm{MHz}$. The primary laser beam is guided through a harmonic generator to create second, third, and fourth harmonics $(517,345$, and $258 \mathrm{~nm}$, respectively). The fourth harmonic is guided to the electron column and focused with an $f=15 \mathrm{~cm}$ lens onto the electron cathode, which is accessible through a vacuum window. Earlier work using the same setup has shown that this photoemission process results in electron pulses with a temporal spread in the picosecond range. ${ }^{46} \mathrm{We}$ use a gradient neutral-density filter to change the fourthharmonic pulse energy from 0 to $1.5 \mathrm{~nJ} /$ pulse, which results in an average number of electrons per pulse up to 400. The corresponding time-averaged beam current on the sample was 0-325 pA measured with a Faraday cup. The error in the current measurement is $\sim 25 \%$, limited by the stability in the laser power and measurement method. In the experiments, the electron spot size has a diameter of $\sim 600 \mathrm{~nm}$. Using the same setup, a higher spatial resolution can be achieved at the expense of lower current on the sample. ${ }^{46}$ All the experiments are performed at room temperature and at a pressure of $10^{-6}$ mbar. 
Laser-Electron Beam Overlap. The second harmonic $(\lambda$ $=517 \mathrm{~nm}$ ) of the same primary laser beam is passed through a linear stage (Newport M-IMS600PP) with motor controller (Newport ESP301-1G), after which it is sent through a pellicle beam splitter (8:92), guided into the SEM sample chamber through a vacuum window, and focused onto the sample to a $\sim 10 \mu \mathrm{m}$ diameter spot using an $\mathrm{Al}$ parabolic mirror $(1.46 \pi \mathrm{sr}$ acceptance angle, 0.1 parabola parameter, and $0.5 \mathrm{~mm}$ focal distance). In the pump-probe measurements the secondharmonic path length was tuned such that the light pulse was delayed $1.3 \mathrm{~ns}$ with respect to the electron pulse. The secondand fourth-harmonic laser powers were independently controlled such that measurements with varying number of electrons per pulse could be done for constant secondharmonic PL power.

$\mathrm{CL}$ and PL Collection. Luminescence from the sample is collected using the $\mathrm{Al}$ parabolic mirror and directed to a light collection and analysis system. Light collected by the mirror is focused $(f=16 \mathrm{~cm})$ onto the entrance facet of a multimode fiber (550 $\mu \mathrm{m}$ core diameter), creating a confocal collection geometry, which limits the PL and CL collection depth in the sample. The fiber guides the light to a Czerny-Turner spectrometer equipped with a CCD array detector (Princeton Spec10) and grating containing 150 lines/mm and blaze wavelength corresponding to $500 \mathrm{~nm}$. A long-pass filter $(\lambda>$ $532 \mathrm{~nm})$ is used to suppress scattered pump laser light in the detection path. TCSPC measurements are performed by sending the CL signal to a single-photon avalanche photodiode (MPD PD-100) analyzed by time correlation (Picoquant PicoHarp 300), which builds a delay histogram. In this case, an additional bandpass filter $(\lambda=650 \pm 75 \mathrm{~nm})$ is used, corresponding to the spectral range within which $\mathrm{NV}$ emission occurs. We use the third-harmonic laser pulse measured with a photodiode as the trigger for the time-correlated measurements. The PL, CL, and PP data in Figure $1 \mathrm{~b}$ and Figure $2 \mathrm{a}$ are collected over a time of $1 \mathrm{~min}$ each. The light collection geometry in this setup typically allows the collection of light within a $20 \times 20 \mu \mathrm{m}^{2}$ area. Only light emitted in this area, and within the escape cone of diamond, can be collected efficiently. Given the critical angle for diamond $\left(\theta_{c}<24.6^{\circ}\right)$, we can estimate that light emitted at a depth down to $20 \mu \mathrm{m}$ inside the diamond can still be collected. Nevertheless, emission beyond this $20 \mu \mathrm{m}$ depth might reach the surface at a position outside of the collection area; thus the collection efficiency decreases at larger depths.

\section{ASSOCIATED CONTENT}

\section{S Supporting Information}

The Supporting Information is available free of charge at https://pubs.acs.org/doi/10.1021/acsphotonics.9b01463.

Additional experimental data and description of the model (PDF)

\section{AUTHOR INFORMATION}

\section{Corresponding Author}

*E-mail: m.sola@amolf.nl.

\section{ORCID}

Magdalena Solà-Garcia: 0000-0002-2614-1050

Toon Coenen: 0000-0002-8043-9798

Albert Polman: 0000-0002-0685-3886

\section{Notes}

The authors declare no competing financial interest.

\section{ACKNOWLEDGMENTS}

This work is part of the research program of AMOLF, which is partly financed by the Dutch Research Council (NWO). This project has received funding from the European Research Council (ERC) under the European Union's Horizon 2020 research and innovation program (grant agreement no. 695343). We gratefully acknowledge the technical support of Erik Kieft, Hans Zeijlemaker, and Dion Ursem. We also gratefully acknowledge discussions with Mayeul Chipaux in the initial phase of this project, Patrick Maletinksy and Elke Neu for providing the bulk diamond sample, and Kévin Cognée for careful reading of the manuscript.

\section{REFERENCES}

(1) Brouri, R.; Beveratos, A.; Poizat, J.-P.; Grangier, P. Photon Antibunching in the Fluorescence of Individual Color Centers in Diamond. Opt. Lett. 2000, 25 (17), 1294-1296.

(2) Doherty, M. W.; Manson, N. B.; Delaney, P.; Jelezko, F.; Wrachtrup, J.; Hollenberg, L. C. L. The Nitrogen-Vacancy Colour Centre in Diamond. Phys. Rep. 2013, 528 (1), 1-45.

(3) Gruber, A.; Dräbenstedt, A.; Tietz, C.; Fleury, L.; Wrachtrup, J.; von Borczyskowski, C. Scanning Confocal Optical Microscopy and Magnetic Resonance on Single Defect Centers. Science (Washington, DC, U. S.) 2012, 276, 2012-2015.

(4) Wee, T.-L.; Tzeng, Y.-K.; Han, C.-C.; Chang, H.-C.; Fann, W.; Hsu, J.-H.; Chen, K.-M.; Yu, Y.-C. Two-Photon Excited Fluorescence of Nitrogen-Vacancy Centers in Proton-Irradiated Type Ib Diamond. J. Phys. Chem. A 2007, 111 (38), 9379-9386.

(5) Berthel, M.; Mollet, O.; Dantelle, G.; Gacoin, T.; Huant, S.; Drezet, A. Photophysics of Single Nitrogen-Vacancy Centers in Diamond Nanocrystals. Phys. Rev. B: Condens. Matter Mater. Phys. 2015, 91, 035308.

(6) Kurtsiefer, C.; Mayer, S.; Zarda, P.; Weinfurter, H. Stable SolidState Source of Single Photons. Phys. Rev. Lett. 2000, 85 (2), 290293.

(7) Hausmann, B. J. M.; Shields, B.; Quan, Q.; Maletinsky, P.; McCutcheon, M.; Choy, J. T.; Babinec, T. M.; Kubanek, A.; Yacoby, A.; Lukin, M. D.; et al. Integrated Diamond Networks for Quantum Nanophotonics. Nano Lett. 2012, 12, 1578-1582.

(8) Aharonovich, I.; Englund, D.; Toth, M. Solid-State SinglePhoton Emitters. Nat. Photonics 2016, 10 (10), 631-641.

(9) Awschalom, D. D.; Hanson, R.; Wrachtrup, J.; Zhou, B. B. Quantum Technologies with Optically Interfaced Solid-State Spins. Nat. Photonics 2018, 12 (9), 516-527.

(10) Balasubramanian, G.; Neumann, P.; Twitchen, D.; Markham, M.; Kolesov, R.; Mizuochi, N.; Isoya, J.; Achard, J.; Beck, J.; Tissler, J.; et al. Ultralong Spin Coherence Time in Isotopically Engineered Diamond. Nat. Mater. 2009, 8 (5), 383-387.

(11) Nemoto, K.; Trupke, M.; Devitt, S. J.; Scharfenberger, B.; Buczak, K.; Schmiedmayer, J.; Munro, W. J. Photonic Quantum Networks Formed from NV ${ }^{-}$Centers. Sci. Rep. 2016, 6 (May), 26284.

(12) Hensen, B.; Bernien, H.; Dréau, A. E.; Reiserer, A.; Kalb, N.; Blok, M. S.; Ruitenberg, J.; Vermeulen, R. F. L.; Schouten, R. N.; Abellán, C.; et al. Loophole-Free Bell Inequality Violation Using Electron Spins Separated by 1.3 Kilometres. Nature 2015, 526, 682686.

(13) Taylor, J. M.; Cappellaro, P.; Childress, L.; Jiang, L.; Budker, D.; Hemmer, P. R.; Yacoby, A.; Walsworth, R.; Lukin, M. D. HighSensitivity Diamond Magnetometer with Nanoscale Resolution. Nat. Phys. 2008, 4 (10), 810-816.

(14) Mamin, H. J.; Kim, M.; Sherwood, M. H.; Rettner, C. T.; Ohno, K.; Awschalom, D. D.; Rugar, D. Nanoscale Nuclear Magnetic Resonance with a Nitrogen-Vacancy Spin Sensor. Science (Washington, DC, U. S.) 2013, 339, 557-561. 
(15) Kucsko, G.; Maurer, P. C.; Yao, N. Y.; Kubo, M.; Noh, H. J.; Lo, P. K.; Park, H.; Lukin, M. D. Nanometre-Scale Thermometry in a Living Cell. Nature 2013, 500 (7460), 54-58.

(16) Kolesov, R.; Grotz, B.; Balasubramanian, G.; Stöhr, R. J.; Nicolet, A. A. L.; Hemmer, P. R.; Jelezko, F.; Wrachtrup, J. WaveParticle Duality of Single Surface Plasmon Polaritons. Nat. Phys. 2009, 5 (7), 470-474.

(17) Aslam, N.; Waldherr, G.; Neumann, P.; Jelezko, F.; Wrachtrup, J. Photo-Induced Ionization Dynamics of the Nitrogen Vacancy Defect in Diamond Investigated by Single-Shot Charge State Detection. New J. Phys. 2013, 15, 013064.

(18) Hacquebard, L.; Childress, L. Charge-State Dynamics during Excitation and Depletion of the Nitrogen-Vacancy Center in Diamond. Phys. Rev. A: At., Mol., Opt. Phys. 2018, 97 (June), 063408. (19) Siyushev, P.; Pinto, H.; Vörös, M.; Gali, A.; Jelezko, F.; Wrachtrup, J. Optically Controlled Switching of the Charge State of a Single Nitrogen-Vacancy Center in Diamond at Cryogenic Temperatures. Phys. Rev. Lett. 2013, 110 (16), 167402.

(20) Fu, K.-M. C.; Santori, C.; Barclay, P. E.; Beausoleil, R. G. Conversion of Neutral Nitrogen-Vacancy Centers to Negatively Charged Nitrogen-Vacancy Centers through Selective Oxidation. Appl. Phys. Lett. 2010, 96, 121907.

(21) Hauf, M. V.; Grotz, B.; Naydenov, B.; Dankerl, M.; Pezzagna, S.; Meijer, J.; Jelezko, F.; Wrachtrup, J.; Stutzmann, M.; Reinhard, F.; et al. Chemical Control of the Charge State of Nitrogen-Vacancy Centers in Diamond. Phys. Rev. B: Condens. Matter Mater. Phys. 2011, 83, 081304.

(22) Rondin, L.; Dantelle, G.; Slablab, A.; Grosshans, F.; Treussart, F.; Bergonzo, P.; Perruchas, S.; Gacoin, T.; Chaigneau, M.; Chang, H.; et al. Surface-Induced Charge State Conversion of NitrogenVacancy Defects in Nanodiamonds. Phys. Rev. B: Condens. Matter Mater. Phys. 2010, 82, 115449.

(23) Grotz, B.; Hauf, M. V.; Dankerl, M.; Naydenov, B.; Pezzagna, S.; Meijer, J.; Jelezko, F.; Wrachtrup, J.; Stutzmann, M.; Reinhard, F.; et al. Charge State Manipulation of Qubits in Diamond. Nat. Commun. 2012, 3, 729.

(24) Kato, H.; Wolfer, M.; Schreyvogel, C.; Kunzer, M.; Müllersebert, W.; Obloh, H.; Yamasaki, S.; Nebel, C. Tunable Light Emission from Nitrogen- Vacancy Centers in Single Crystal Diamond PIN Diodes. Appl. Phys. Lett. 2013, 102, 151101.

(25) Tizei, L. H. G.; Kociak, M. Spectrally and Spatially Resolved Cathodoluminescence of Nanodiamonds: Local Variations of the $\mathrm{NV}^{0}$ Emission Properties. Nanotechnology 2012, 23, 175702.

(26) Tizei, L. H. G.; Kociak, M. Spatially Resolved Quantum NanoOptics of Single Photons Using an Electron Microscope. Phys. Rev. Lett. 2013, 110 (15), 153604.

(27) Lourenço-Martins, H.; Kociak, M.; Meuret, S.; Treussart, F.; Lee, Y. H.; Ling, X. Y.; Chang, H.-C.; Galvão Tizei, L. H. Probing Plasmon-NV $\mathrm{NV}^{0}$ Coupling at the Nanometer Scale with Photons and Fast Electrons. ACS Photonics 2018, 5, 324-328.

(28) Kumar, S.; Huck, A.; Andersen, U. L. Efficient Coupling of a Single Diamond Color Center to Propagating Plasmonic Gap Modes. Nano Lett. 2013, 13, 1221-1225.

(29) Egerton, R. F. Electron Energy-Loss Spectroscopy in the TEM. Rep. Prog. Phys. 2009, 72 (1), 016502.

(30) Klein, C. A. Radiation Ionization Energies in Semiconductors: Speculations about the Role of Plasmons. Proc. Int. Conf. Phys. Semicond. 1966, 21, 307-311.

(31) Kociak, M.; Zagonel, L. F. Cathodoluminescence in the Scanning Transmission Electron Microscope. Ultramicroscopy 2017, $174,50-69$.

(32) Watanabe, H.; Kitamura, T.; Nakashima, S.; Shikata, S. Cathodoluminescence Characterization of a Nitrogen-Doped Homoepitaxial Diamond Thin Film. J. Appl. Phys. 2009, 105 (2009), 093529.

(33) Zhang, H.; Glenn, D. R.; Schalek, R.; Lichtman, J. W.; Walsworth, R. L. Efficiency of Cathodoluminescence Emission by Nitrogen-Vacancy Color Centers in Nanodiamonds. Small 2017, 13, 1700543.
(34) Davies, G. Dynamic Jahn-Teller Distortions at Trigonal Optical Centres in Diamond. J. Phys. C: Solid State Phys. 1979, 12 (13), 2551-2566.

(35) Tallaire, A.; Collins, A. T.; Charles, D.; Achard, J.; Sussmann, R.; Gicquel, A.; Newton, M. E.; Edmonds, A. M.; Cruddace, R. J. Characterisation of High-Quality Thick Single-Crystal Diamond Grown by CVD with a Low Nitrogen Addition. Diamond Relat. Mater. 2006, 15, 1700-1707.

(36) Robins, L. H.; Cook, L. P.; Farabaugh, E. N.; Feldman, A. Cathodoluminescence of Defects in Diamond Films and Particles Grown by Hot-Filament Chemical-Vapor Deposition. Phys. Rev. B: Condens. Matter Mater. Phys. 1989, 39 (18), 368-377.

(37) Malykhin, S. A.; Houard, J.; Ismagilov, R. R.; Orekhov, A. S.; Vella, A.; Obraztsov, A. N. Luminescent Characteristics of NeedleLike Single Crystal Diamonds. Phys. Status Solidi B 2018, 255, 1700189.

(38) Barwick, B.; Flannigan, D. J.; Zewail, A. H. Photon-Induced near-Field Electron Microscopy. Nature 2009, 462 (7275), 902-906.

(39) Feist, A.; Echternkamp, K. E.; Schauss, J.; Yalunin, S. V.; Schäfer, S.; Ropers, C. Quantum Coherent Optical Phase Modulation in an Ultrafast Transmission Electron Microscope. Nature 2015, 521 (7551), 200-203.

(40) Rubiano Da Silva, N.; Möller, M.; Feist, A.; Ulrichs, H.; Ropers, C.; Schäfer, S. Nanoscale Mapping of Ultrafast Magnetization Dynamics with Femtosecond Lorentz Microscopy. Phys. Rev. X 2018, 8 (3), 31052.

(41) Liao, B.; Najafi, E. Scanning Ultrafast Electron Microscopy: A Novel Technique to Probe Photocarrier Dynamics with High Spatial and Temporal Resolutions. Mater. Today Phys. 2017, 2, 46-53.

(42) Merano, M.; Sonderegger, S.; Crottini, A.; Collin, S.; Renucci, P.; Pelucchi, E.; Malko, A.; Baier, M. H.; Kapon, E.; Deveaud, B.; et al. Probing Carrier Dynamics in Nanostructures by Picosecond Cathodoluminescence. Nature 2005, 438 (7067), 479-482.

(43) Feist, A.; Bach, N.; Rubiano da Silva, N.; Danz, T.; Möller, M.; Priebe, K. E.; Domröse, T.; Gatzmann, J. G.; Rost, S.; Schauss, J.; et al. Ultrafast Transmission Electron Microscopy Using a LaserDriven Fi Eld Emitter: Femtosecond Resolution with a High Coherence Electron Beam. Ultramicroscopy 2017, 176, 63-73.

(44) Shahmohammadi, M.; Ganière, J.-D.; Zhang, H.; Ciechonski, R.; Vescovi, G.; Kryliouk, O.; Tchernycheva, M.; Jacopin, G. Excitonic Diffusion in InGaN/GaN Core-Shell Nanowires. Nano Lett. 2016, $16,243-249$.

(45) Carbone, F.; Barwick, B.; Kwon, O.-H.; Park, H. S.; Baskin, J. S.; Zewail, A. H. EELS Femtosecond Resolved in 4D Ultrafast Electron Microscopy. Chem. Phys. Lett. 2009, 468, 107-111.

(46) Meuret, S.; Solà Garcia, M.; Coenen, T.; Kieft, E.; Zeijlemaker, H.; Lätzel, M.; Christiansen, S.; Woo, S. Y.; Ra, Y. H.; Mi, Z.; et al. Complementary Cathodoluminescence Lifetime Imaging Configurations in Scanning Electron Microscopy. Ultramicroscopy 2019, 197, $28-38$.

(47) Sun, J.; Melnikov, V. A.; Khan, J. I.; Mohammed, O. F. RealSpace Imaging of Carrier Dynamics of Materials Surfaces by SecondGeneration Four-Dimensional Scanning Ultrafast Electron Microscopy. J. Phys. Chem. Lett. 2015, 6, 3884-3890.

(48) Krishnamurti, D. The Raman Spectrum of Diamond. Proc. Indian Acad. Sci., Sect. A 1954, 40 (5), 211-216.

(49) Zaitsev, A. M. Optical Properties of Diamond: A Data Handbook; Springer, 2001.

(50) Iakoubovskii, K.; Adriaenssens, G. J. Luminescence Excitation Spectra in Diamond. Phys. Rev. B: Condens. Matter Mater. Phys. 2000, 61 (15), 10174-10182.

(51) Demers, H.; Poirier-Demers, N.; Couture, A. R.; Joly, D.; Guilmain, M.; de Jonge, N.; Drouin, D. Three-Dimensional Electron Microscopy Simulation with the CASINO Monte Carlo Software. Scanning 2011, 33 (April), 135-146.

(52) Beha, K.; Batalov, A.; Manson, N. B.; Bratschitsch, R.; Leitenstorfer, A. Optimum Photoluminescence Excitation and Recharging Cycle of Single Nitrogen-Vacancy Centers in Ultrapure Diamond. Phys. Rev. Lett. 2012, 109 (9), 097404. 
(53) Manson, N. B.; Harrison, J. P. Photo-Ionization of the Nitrogen-Vacancy Center in Diamond. Diamond Relat. Mater. 2005, 14 (10), 1705-1710.

(54) Hopper, D. A.; Grote, R. R.; Exarhos, A. L.; Bassett, L. C. NearInfrared-Assisted Charge Control and Spin Readout of the NitrogenVacancy Center in Diamond. Phys. Rev. B: Condens. Matter Mater. Phys. 2016, 94, 241201.

(55) Lu, H.-C.; Peng, Y.-C.; Chou, S.-L.; Lo, J.-I.; Cheng, B.-M.; Chang, H.-C. Far-UV-Excited Luminescence of Nitrogen-Vacancy Centers: Evidence for Diamonds in Space. Angew. Chem., Int. Ed. 2017, 56 (46), 14469-14473.

(56) Mizuochi, N.; Makino, T.; Kato, H.; Takeuchi, D.; Ogura, M.; Okushi, H.; Nothaft, M.; Neumann, P.; Gali, A.; Jelezko, F.; et al. Electrically Driven Single-Photon Source at Room Temperature in Diamond. Nat. Photonics 2012, 6 (May), 299-303.

(57) Lohrmann, A.; Pezzagna, S.; Dobrinets, I.; Spinicelli, P.; Jacques, V.; Roch, J.-F.; Meijer, J.; Zaitsev, A. M. Diamond Based Light-Emitting Diode for Visible Single-Photon Emission at Room Temperature. Appl. Phys. Lett. 2011, 99, 251106.

(58) Malinauskas, T.; Jarasiunas, K.; Ivakin, E.; Ralchenko, V.; Gontar, A.; Ivakhnenko, S. Optical Evaluation of Carrier Lifetime and Diffusion Length in Synthetic Diamonds. Diamond Relat. Mater. 2008, $17,1212-1215$

(59) Shimomura, T.; Kubo, Y.; Barjon, J.; Tokuda, N.; Akimoto, I.; Naka, N. Quantitative Relevance of Substitutional Impurities to Carrier Dynamics in Diamond. Phys. Rev. Mater. 2018, 2, 1-7. 